\title{
Analysis of heart rate variability in a rat model of induced pulmonary hypertension
}

\author{
Hernâni Gonçalves a,*, Tiago Henriques-Coelho ${ }^{b}$, João Bernardes ${ }^{c, d}$, \\ Ana Paula Rocha ${ }^{\mathrm{e}, \mathrm{f}}$, Ana Brandão-Nogueira ${ }^{\mathrm{b}}$, Adelino Leite-Moreira ${ }^{\mathrm{b}}$ \\ a Departamento de Geociências, Ambiente e Ordenamento do Território, Faculdade de Ciências, Universidade do Porto, Rua do Campo Alegre 687, 4169-007 Porto, Portugal \\ b Servico de Fisiologia, Faculdade de Medicina, Universidade do Porto, Alameda Prof. Hernâni Monteiro, 4200-319 Porto, Portugal \\ c Departamento de Ginecologia e Obstetrícia, Faculdade de Medicina, Universidade do Porto, Alameda Prof. Hernâni Monteiro, $4200-319$ Porto, Portugal \\ d Instituto Nacional de Engenharia Biomédica (INEB), Porto, Portugal \\ e Departamento de Matemática, Faculdade de Ciências, Universidade do Porto, Rua do Campo Alegre 687, 4169-007 Porto, Portugal \\ ${ }^{\mathrm{f}}$ Centro de Matemática da Universidade do Porto, Porto, Portugal
}

\section{A R T I C L E I N F O}

\section{Article history:}

Received 21 August 2009

Received in revised form 13 March 2010

Accepted 25 April 2010

\section{Keywords:}

Heart rate variability (HRV)

Entropy

Time and frequency domain analysis

Wistar rats

Ketamine

Monocrotaline

\begin{abstract}
A B S T R A C T
Monocrotaline (MCT) is commonly used to experimentally induce pulmonary hypertension (PH), which might lead to chronic heart failure. In this study, linear and non-linear heart rate (HR) dynamics were weekly assessed in MCT-treated and non-treated Wistar rats.

The HR of 10 adult Wistar rats injected with MCT (MCT group) and of 10 similar rats injected with vehicle (non-MCT group), anesthetized with Ketamine, was weekly recorded during 4 weeks. The first four segments of 1-min length of each HR recording were analysed using linear, time and frequency domains, and approximate (ApEn) and sample (SampEn) entropy indices, considering recently proposed values for the threshold parameter of ApEn and SampEn. Statistical analysis was performed using 95\% confidence intervals and statistical tests.

Along the study period, an overall weekly maintenance of HR indices, or a decrease, namely in weeks 1-2, was manifest, in the MCT group, except for LF and LF/HF, in week 1, denoting a short-term increase in sympathetic activity without any other changes. On the other hand, a maintenance of HR indices, or an increase, namely on week 4, was observed in the non-MCT group, except for LF/HF, denoting a long-term increase of the overall activity of HR control systems, with a parasympathetic like dominance.

Studies on long-term HR dynamics should be performed in very carefully controlled experimental settings, as significant weekly changes may occur, both among anesthetized MCT-treated and non-treated rats.

(C) 2010 IPEM. Published by Elsevier Ltd. All rights reserved.
\end{abstract}

\section{Introduction}

The limitations related to the research on some human diseases, particularly during fetal life, lead to the need of alternatives, such as experimental studies on animal models. This is a scenario that may be found regarding heart rate (HR) analysis, on the study of diseases such as pulmonary hypertension (PH) and right cardiac failure, not only during post natal, but also during pre-natal life, as it happens in fetal growth restriction conditions associated to left and right ventricular increased afterload, progressing to tricuspid valve failure and abnormal ductus venosus blood flow [25].
Monocrotaline (MCT) is a plant alkaloid that produces pulmonary endothelial damage and edema. It is used to produce an acute model of non-cardiogenic pulmonary edema and a chronic model of cardiac failure associated to pulmonary hypertension with increased right ventricular end-systolic and end-diastolic volumes arising even under non-failing conditions [17,24]. A direct cardiotoxic effect, associated with myocarditis and coronary arteriolar medial thickening, has also been described and may further account for a depression of ventricular function [2]. MCT administration to rats has produced contradictory effects on heart rate (HR) dynamics, either producing an increase [33] or a decrease in its mean value [17]. However, studies on short- and long-term effects of MCT on complex HR dynamics are still lacking.

The objective of this study was the assessment of short- and long-term linear and non-linear HR indices in MCT-treated and non-treated Wistar rats, regarding a better understanding of the pathophysiological meaning of those indices and a better knowledge of their role in HR research.

\footnotetext{
* Corresponding author. Tel.: +351 220402240.

E-mail addresses: hernani.goncalves@fc.up.pt (H. Gonçalves), joaobern@med.up.pt (J. Bernardes).
} 


\section{Methods}

\subsection{Animal model}

Animal experiments were performed according to the Portuguese law for animal welfare and the National Institutes of Health Guide for the Care and Use of Laboratory Animals (NIH Pub. No. 8523, Revised 1996). Twenty adult female Wistar rats (Charles River Laboratories; Barcelona, Spain), weighing 215-260 g, and with ages ranging between 6 and 8 weeks were selected. They were housed in groups of 5 rats/cage, in a controlled environment under a 12:12-h light-dark cycle, at a room temperature of $22^{\circ} \mathrm{C}$, with a free supply of food and water. Of these rats, 10 were treated with $60 \mathrm{mg} / \mathrm{kg}$ of MCT (Sigma, Barcelona, Spain; MCT group) and 10 with a similar volume $(2 \mathrm{~mL} / \mathrm{kg}$ ) of the vehicle (non-MCT group). MCT was administrated by subcutaneous injection. All rats were initially ECG monitored during 20 min (week 0) under ketamine anaesthesia $(50 \mathrm{mg} / \mathrm{kg}$ ) and subsequently monitored following a similar recording protocol for 4 weeks (weeks 1-4).

\subsection{Heart rate acquisition and pre-processing}

Four electrodes were placed subcutaneously, one in each leg, to acquire the ECG signal at a sampling rate of $500 \mathrm{~Hz}$, according to a standardized and previously tested procedure [14]. The tachogram was obtained after automatic R wave detection in the ECG signal and posterior expert validation. Subsequently, it was resampled at a frequency of $8 \mathrm{~Hz}$ (an adequate rate regarding the spectral bands of interest) and converted to the HR signal, in beats per minute (bpm) using cubic spline interpolation.

\subsection{Linear and non-linear heart rate analysis}

The first four segments of 1-min length of each HR recording with good signal quality (signal loss events were manually identified) were selected for the analysis, excluding the first 2 min in order to assure a stable tracing (an example is shown in Fig. 1). The selected segments were analysed with common time and frequency domain measures, elsewhere described in detail
[35,11]. Time domain measures included mean HR (mHR), HR standard-deviation (sdHR), long-term irregularity (LTI), the mean amplitude between the maximum and minimum for each minute $(\Delta)$, short-term variability (STV) and interval index (II). For spectral characterization of the data, non-parametric (Welch) spectrum estimation was performed [37], considering a Hanning window of 256 points and $62.5 \%$ of superposition. The decomposition of HR variability allows the identification of a very low frequency component (VLF), below $0.04 \mathrm{~Hz}$, and two components associated to the low and high frequencies (LF and HF), centered approximately at $0.1 \mathrm{~Hz}(0.04-1.00 \mathrm{~Hz})$ and at the respiratory frequency $(1.00-3.00 \mathrm{~Hz})$, respectively [18]. In order to validate the considered spectral bands, the rats' respiration cycles were visually observed and counted during $1 \mathrm{~min}$, on the last two weeks of the MCT group.

For non-linear analysis, ApEn and SampEn were used as in other previous human and rat studies [14,31]. For approximate entropy [29], $\operatorname{ApEn}(m, r)$, and sample entropy [32], $\operatorname{SampEn}(m, r)-$ which are non-linear measures obtained through direct signal estimation, capable of quantifying signal complexity (or irregularity) robustly with short segments - the parameter $m$ is the embedding dimension and the parameter $r$ acts as a threshold. The considered estimator for $\operatorname{ApEn}(m, r)$, based on $N$ signal points, was

$$
\begin{aligned}
\operatorname{ApEn}(m, r, N)= & \phi^{m}(r)-\phi^{m+1}(r)=\frac{1}{N-m+1} \sum_{i=1}^{N-m+1} \ln \left(C_{r}^{m}(i)\right) \\
& -\frac{1}{N-m} \sum_{i=1}^{N-m} \ln \left(C_{r}^{m+1}(i)\right)
\end{aligned}
$$

SampEn is computed in a similar manner as ApEn, with the major difference that SampEn does not count self-matches, having reduced bias when compared to ApEn. The values $0.1 \mathrm{SD}, 0.15$ SD and 0.2 SD were initially considered for the parameter $r$, while $m$ was considered as 2 [29]. These values for $r$ and $m$ produce good statistical validity of $\operatorname{ApEn}(m, r)$, based on calculations that included both theoretical analysis and clinical applications [30].

Three other values for parameter " $r$ " different from what has been broadly considered in the analysis of the fetal heart rate (FHR) signal were considered in this work, as previously proposed [14].
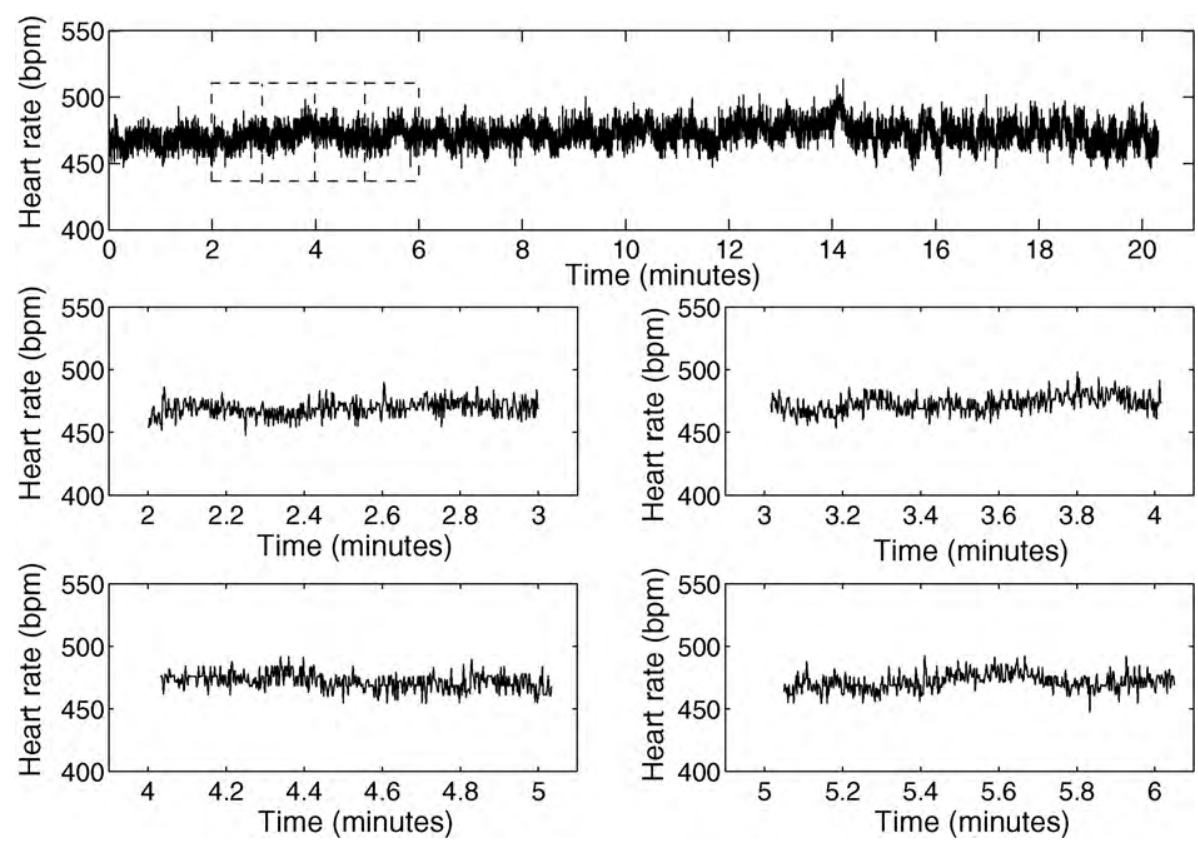

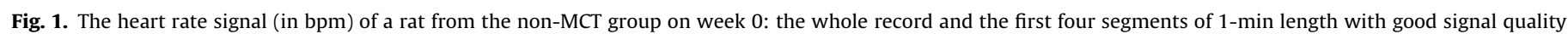
(excluding the first $2 \mathrm{~min}$ ). 
Table 1

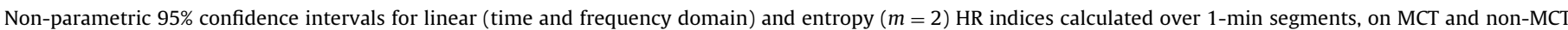

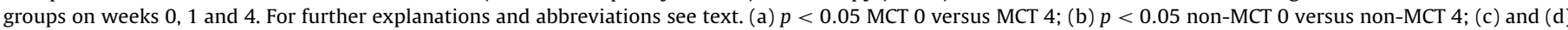
$p<0.05$ Null hypothesis assuming that all 5 weeks have identical median for the MCT and non-MCT groups, respectively.

\begin{tabular}{|c|c|c|c|c|c|c|c|}
\hline \multirow{2}{*}{$\begin{array}{l}\text { HR } \\
\text { indices }\end{array}$} & \multicolumn{3}{|c|}{ MCT group $(n=10)$} & \multicolumn{3}{|c|}{ non-MCT group $(n=10)$} & \multirow[t]{2}{*}{$p$} \\
\hline & Week 0 & Week 1 & Week $4^{\mathrm{a}}$ & Week $0^{\mathrm{a}}$ & Week $1^{\text {b }}$ & Week 4 & \\
\hline $\mathrm{mHR}$ & $404-443$ & $367-411$ & $399-446$ & $458-489$ & $462-479$ & $475-492$ & $\mathrm{~d}$ \\
\hline sdHR & $6.8-8.8$ & $6.8-10.0$ & 6.9-9.3 & $6.9-8.1$ & $6.6-9.2$ & $9.4-10.9$ & $\mathrm{~b}, \mathrm{~d}$ \\
\hline LTI & $11.1-14.5$ & $11.7-16.2$ & $9.9-12.9$ & $10.0-11.9$ & $9.0-12.7$ & $11.4-15.9$ & \\
\hline$\Delta$ & $36.9-52.7$ & $35.1-50.9$ & $38.2-50.6$ & $42.1-51.0$ & $42.3-56.0$ & $52.5-70.0$ & $b, d$ \\
\hline STV & $4.6-6.5$ & $4.1-5.5$ & $4.5-7.9$ & $6.8-7.9$ & $6.2-8.4$ & $8.8-11.3$ & $b, d$ \\
\hline II & $0.66-0.83$ & $0.56-0.81$ & $0.79-1.00$ & $0.94-1.09$ & $0.90-1.01$ & $0.96-1.11$ & $a, c, d$ \\
\hline TP & $30.9-44.3$ & $21.6-51.5$ & $27.0-61.9$ & $40.2-54.0$ & $41.2-61.4$ & $65.4-105.9$ & $\mathrm{~b}, \mathrm{c}, \mathrm{d}$ \\
\hline LF & $3.8-8.3$ & $6.6-11.9$ & $6.4-11.8$ & $9.1-13.2$ & $5.9-10.3$ & $8.6-14.5$ & $c, d$ \\
\hline $\mathrm{HF}$ & $12.2-28.0$ & $8.5-10.8$ & $14.3-30.9$ & $19.5-30.2$ & $23.9-35.4$ & $33.3-55.9$ & $\mathrm{~b}, \mathrm{c}, \mathrm{d}$ \\
\hline $\mathrm{LF} / \mathrm{HF}$ & $0.18-0.35$ & $0.48-1.35$ & $0.31-0.59$ & $0.44-0.63$ & $0.19-0.34$ & $0.23-0.39$ & $\mathrm{a}, \mathrm{b}, \mathrm{c}, \mathrm{d}$ \\
\hline $\operatorname{ApEn}(0.1)$ & $0.78-0.81$ & $0.77-0.85$ & $0.78-0.80$ & $0.76-0.79$ & $0.76-0.79$ & $0.76-0.79$ & $\mathrm{~d}$ \\
\hline ApEn (0.15) & $1.00-1.05$ & $0.97-1.04$ & $1.04-1.09$ & $1.04-1.07$ & $1.04-1.09$ & $1.07-1.11$ & $a, b, c, d$ \\
\hline ApEn (0.2) & $1.09-1.18$ & $1.05-1.15$ & $1.16-1.23$ & $1.21-1.26$ & $1.19-1.29$ & $1.27-1.30$ & $\mathrm{a}, \mathrm{b}, \mathrm{c}, \mathrm{d}$ \\
\hline SampEn (0.1) & $1.33-1.62$ & $1.15-1.62$ & $1.43-1.76$ & $1.66-1.93$ & $1.66-1.96$ & $2.01-2.18$ & $\mathrm{~b}, \mathrm{c}, \mathrm{d}$ \\
\hline SampEn (0.15) & $1.27-1.49$ & $1.10-1.49$ & $1.35-1.69$ & $1.59-1.79$ & $1.53-1.94$ & $1.81-2.07$ & $\mathrm{~b}, \mathrm{c}, \mathrm{d}$ \\
\hline SampEn (0.2) & $1.22-1.40$ & $1.05-1.39$ & $1.32-1.60$ & $1.56-1.70$ & $1.49-1.77$ & $1.73-1.95$ & $\mathrm{~b}, \mathrm{c}, \mathrm{d}$ \\
\hline ApEn (0.6) & $0.90-1.04$ & $0.59-1.01$ & $0.96-1.11$ & $1.10-1.15$ & $1.06-1.16$ & $1.10-1.14$ & $\mathrm{c}, \mathrm{d}$ \\
\hline ApEn (0.9) & $0.50-0.74$ & $0.31-0.65$ & $0.60-0.77$ & $0.77-0.81$ & $0.73-0.82$ & $0.75-0.80$ & $c, d$ \\
\hline ApEn (1.2) & $0.33-0.49$ & $0.18-0.38$ & $0.42-0.52$ & $0.51-0.53$ & $0.49-0.56$ & $0.52-0.55$ & $c, d$ \\
\hline SampEn (0.6) & $0.79-1.00$ & $0.53-0.95$ & $0.87-1.06$ & $1.04-1.11$ & $0.98-1.11$ & $1.03-1.11$ & $c, d$ \\
\hline SampEn (0.9) & $0.45-0.66$ & $0.28-0.58$ & $0.51-0.69$ & $0.68-0.75$ & $0.67-0.73$ & $0.67-0.73$ & $\mathrm{c}$ \\
\hline SampEn (1.2) & $0.28-0.44$ & $0.16-0.34$ & $0.35-0.46$ & $0.43-0.47$ & $0.44-0.48$ & $0.45-0.49$ & $\mathrm{c}, \mathrm{d}$ \\
\hline
\end{tabular}

a $n=9$.

b $n=8$.

This choice was related to the fact that the quotient between the coefficient of variation of the FHR and the coefficient of variation of the rat HR signal is about 6. Although entropy indices (ApEn and SampEn) are normalized by the standard-deviation (SD), as the variation about the mean HR signal in rats is about 6 times lower than for FHR, when we are using values 0.1 SD, 0.15 SD and 0.2 SD for parameter " $r$ ", we are considering respectively $10 \%, 15 \%$ and $20 \%$ of a much lower variation around the mean than for the FHR signal. Therefore, we also considered the values 0.6 SD, 0.9 SD and 1.2 SD for parameter " $r$ ".

The segments length, $N$, considered for ApEn and SampEn computation were equal to the number of points on each 1-min analysed segment, which is 480 points after cubic spline interpolation of the HR signal at $8 \mathrm{~Hz}$. The comparison of these measures regarding this procedure is coherent with previously reported results [14].

For the assessment of the comparability between the MCT and non-MCT groups, the baseline characteristics of the rats were analysed, namely regarding the HR indices, before and after exclusion of one rat from each group presenting outlying HR values in the upper and lower range limits. It was also performed an analysis of the results after normalization by subtracting the week 0 value of each measure to the remaining weeks results.

For statistical inference, the Lilliefors test was used to assess normality of the results distributions [9]. Accordingly, parametric and non-parametric bootstrap 95\% confidence intervals (for the mean and median, respectively) [23], as well as the statistical tests $t$-test for two independent samples and ANOVA, or non-parametric Mann-Whitney and Kruskal-Wallis, respectively, were used when appropriate [9].

\section{Results}

The median final rat weight was lower in the MCT group than in the non-MCT group ( $253 \mathrm{~g}$ versus $275 \mathrm{~g}$ ). At the end of third week the rats injected with MCT presented signs of overt HF, namely, lethargy, labored breathing, cachexia, vein and liver engorgement, pleural effusion, and ascites. One rat from the MCT group died in week 3 during instrumentation and two rats died in the non-MCT group after the anaesthetic injection on week 3. For technical reasons, it was not possible to recover one tracing from the non-MCT group in week 0 .

Validation of the spectral bands considered in this study, by visual respiration cycles counting, provided respiration rates ranging from $1.28 \mathrm{~Hz}$ to $2.0 \mathrm{~Hz}$, with average of $1.73 \mathrm{~Hz}$ and standard-deviation of $0.22 \mathrm{~Hz}$, in agreement with the considered bands.

The normality assumption was tested for all combinations of weeks/measures through Lilliefors test. Since the $p$-value was greater than 0.05 for most combinations, a non-parametric approach has been considered: bootstrap percentile 95\% confidence intervals for the median, as well as non-parametric Mann-Whitney and Kruskal-Wallis statistical tests were used.

Unexpectedly, some basal values of HR indices were different in the MCT and non-MCT groups, although they had been selected among similar rats, both before (Table 1 and Figs. 2-5) and after exclusion of one rat from each group, presenting outlying HR values. The groups could not, therefore, be considered equivalent and they were analysed separately and not directly compared between each other.

As shown in Table 1 and illustrated in Figs. 2-5, the MCT group did not evidence significant differences between weeks 0 and 4 for all indices, with the exception of II, $\operatorname{LF} / \mathrm{HF}, \operatorname{ApEn}(2,0.15)$ and $\operatorname{ApEn}(2,0.2)$. Nevertheless, significant variations across the weeks of the experiment can be observed, in particular regarding frequency and entropy indices (Figs. 3-5). Moreover, along the study period, an overall weekly maintenance of HR indices, or a decrease, namely in weeks 1-2, was manifest, in the MCT group, except for LF and LF/HF, in week 1 (Figs. 2-5). On the other hand, a maintenance of HR indices, or an increase, namely on week 4, was observed in the non-MCT group, except for LF/HF (Figs. 2-5).

With respect to the non-MCT group, beyond a significant difference across the weeks of the experiment for most of the considered HRV indices, the difference between weeks 0 and 4 was found to be 

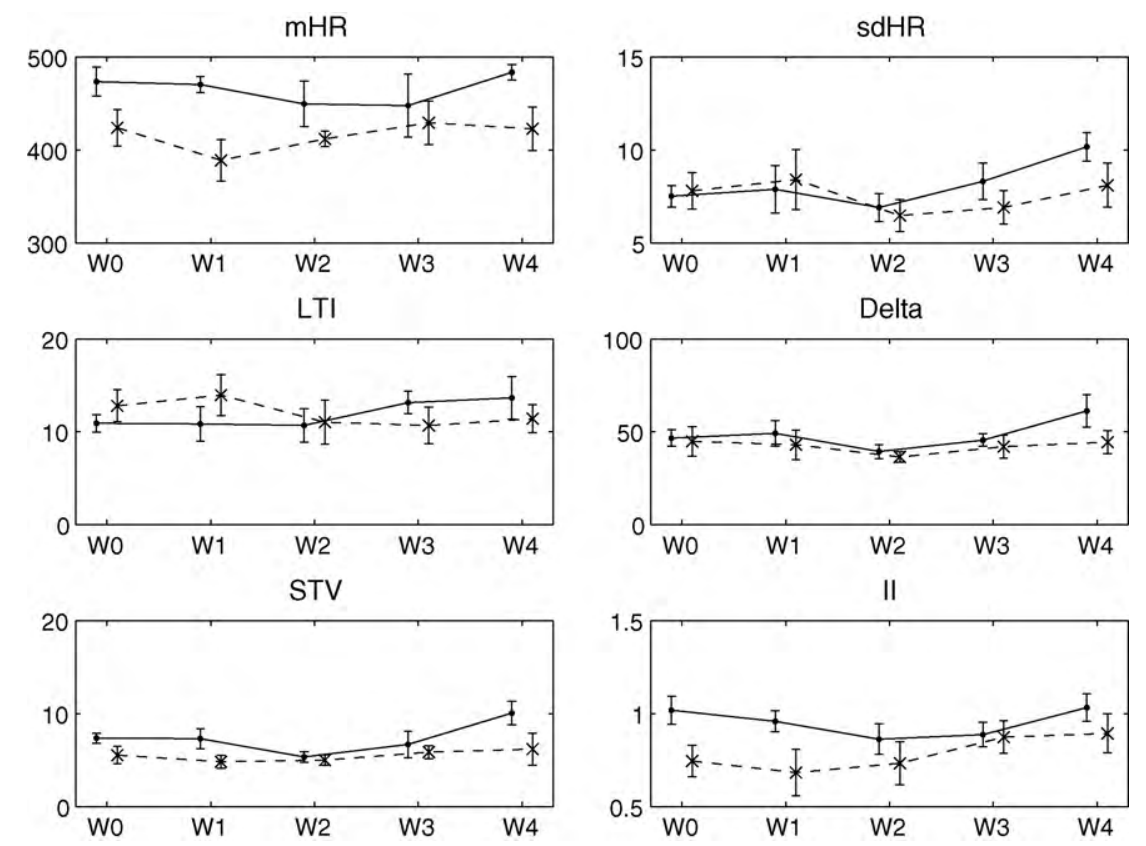

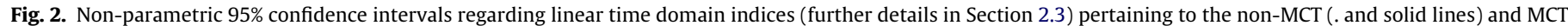
( $\mathrm{x}$ and dashed lines) groups, along the study period.
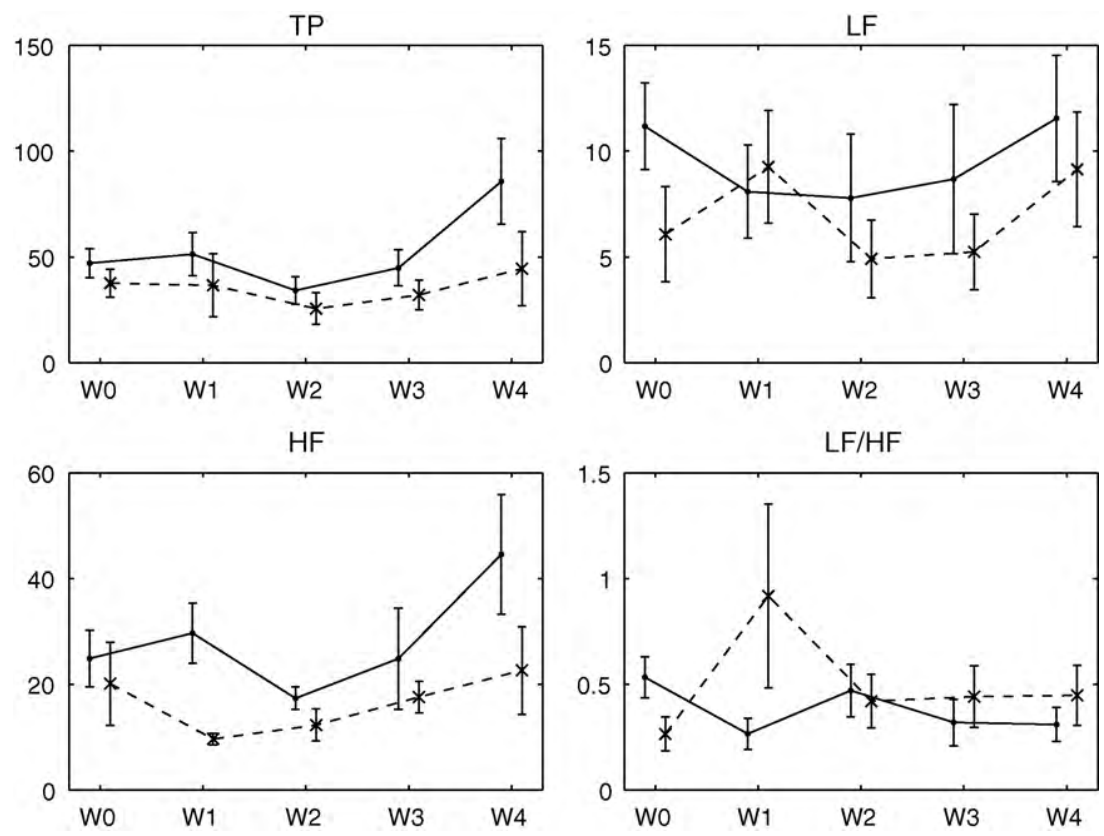

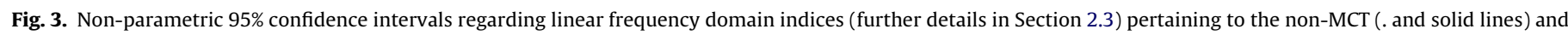
MCT ( $\mathrm{x}$ and dashed lines) groups, along the study period.

significant to the majority of the indices, with the exception of all entropy indices using the recently proposed values for parameter " $r$ ". Furthermore, as illustrated in Fig. 5, a similar behavior across the weeks of protocol was observed for the new three considered values of parameter " $r$ ". Additionally, these results were also found regarding the MCT group (Table 1 and Fig. 5).

\section{Discussion}

In this study linear and non-linear HR dynamics in MCT-treated and non-treated Wistar rats were assessed, regarding a better understanding of those indices and a better knowledge of their role in HR research. To our knowledge, such a study has never been reported. The used rat model has been extensively studied and biochemically, physiologically and histo-pathologically characterized by our group $[10,15,16,20]$. It is of particular interest in experimental studies on pulmonary hypertension and right ventricular heart failure $[2,15,17,20,24,33]$, but it may also help to understand some human fetal pathological conditions associated to progressive heart failure and growth restriction, characterized by left and right ventricular increased afterload, progressing to right heart failure, with tricuspid valve failure and abnormal ductus venosus blood flow [25]. This conforms with the increasing interest of pre and post natal medicine regarding linear and non-linear HR analysis $[1,5,12,13,29,34,35]$. 

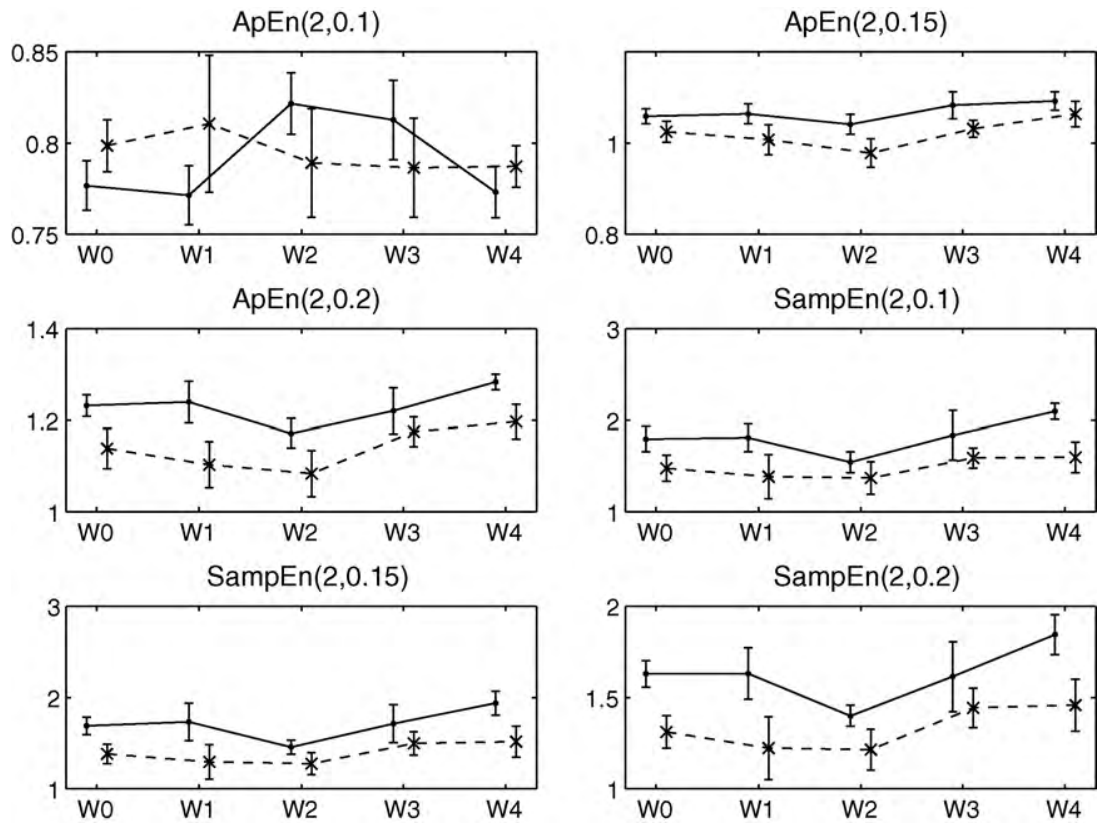

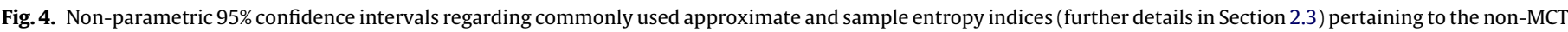
(. and solid lines) and MCT ( $\mathrm{x}$ and dashed lines) groups, along the study period.

To minimize intra- and inter-rats variations in HR indices and make observations more reproducible, the first four segments of 1-min length of each HR recording with good signal quality were selected for the analysis, excluding the first $2 \mathrm{~min}$, in order to assure a stable tracing. In spite of this, large variations were still observed along the study period. Why such variations within relatively homogeneous groups of rats, housed and instrumented similarly? In spite of the efforts to standardize rats selection and treatment, this may have not been enough considering the marked and complex sensitivity of rats behavior to even small environment changes [6]. This was probably worsened because female young adult rats prone to estrus cycle variations were used, because they were preferred at our laboratory, as they seemed to be more sensitive to MCT than males. Another aspect that probably contributed to the large intra- and inter-rat variations, that should be considered in future studies, was the type of instrumentation used to record $\mathrm{HR}$, implying anesthesia with Ketamine and rat manipulation. Ketamine induces a state of sedation, immobility, amnesia and marked analgesia. At pulmonary level, ketamine has no effects in pulmonary vascular resistance. It induces an increase in arterial blood pressure, cardiac output and heart rate. This effect is attributed to an increase in sympathetic activity [22]. Hopefully, this should be substituted by telemetric HR recording avoiding anesthesia and minimizing artificial rat manipulation and instru-
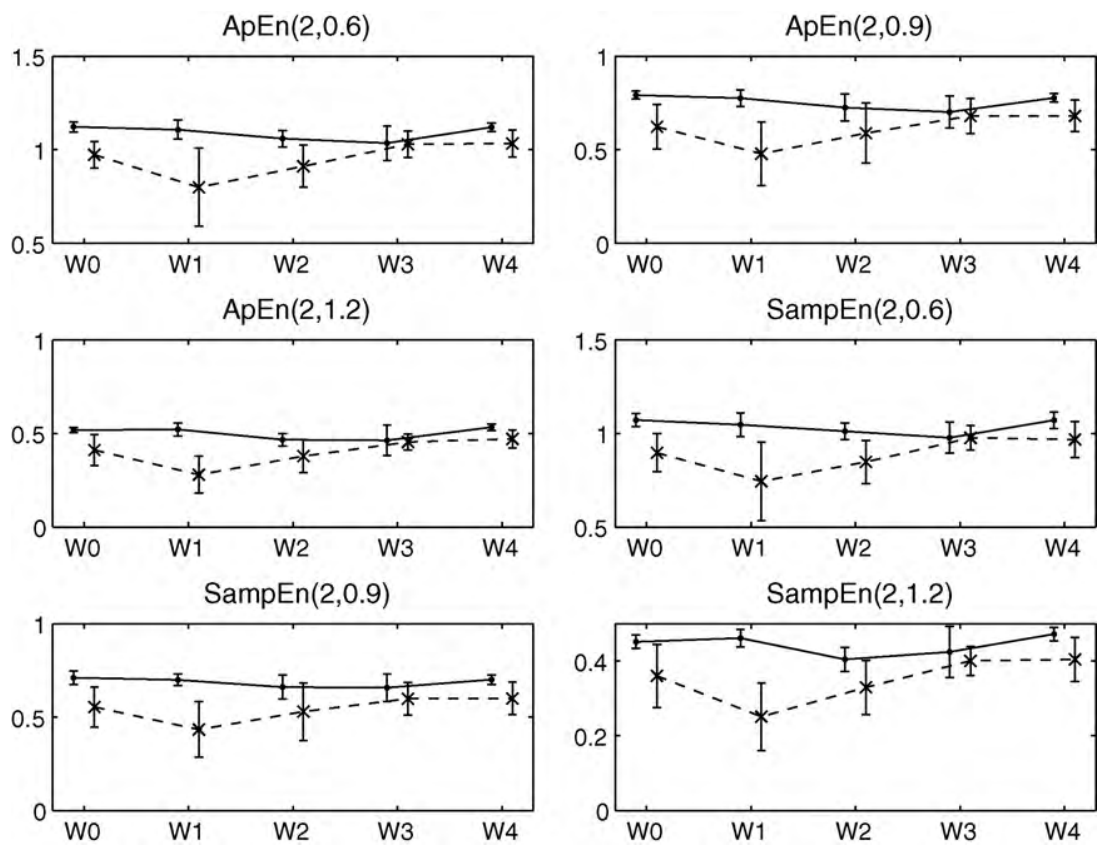

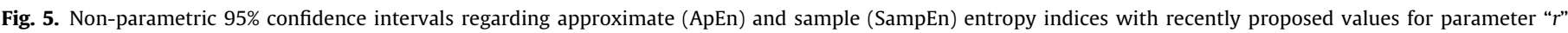
(further details in Section 2.3), pertaining to the non-MCT (. and solid lines) and MCT ( $\mathrm{x}$ and dashed lines) groups, along the study period. 
mentation. All these aspects should be kept in mind and carefully controlled in studies involving long-term observation of rat variables related to behavioral aspects.

In relation to the above discussed issues, unexpectedly, some basal values of HR indices were different in the MCT and non-MCT groups, although they had been selected among similar rats, both before and after exclusion of one rat from each group, presenting outlying HR values. The groups could not, therefore, be considered equivalent and they were analysed separately and not directly compared with each other. This may had arisen not only because of all the aspects discussed before about the extreme individual and environment rat sensitivity, but also because an arbitrary rather than a strict randomized assignment of rats to each of the studied groups was not followed. This is an important study limitation, but reinforces, at the same time, the message that studies on HR dynamics should be performed in very strictly controlled experimental settings.

The dose of MCT $(60 \mathrm{mg} / \mathrm{kg})$ used was associated at 4 weeks with scarce signs of cardiac failure and low mortality, in agreement with other reports $[10,15-17]$. At the end of the study, rats were still used for experiments on acute hypoxia and anoxia, recently reported elsewhere [14]. Anyway, injection of MCT in week 0 was associated with growth restriction and overall weekly maintenance of HR indices, or a decrease, namely in weeks 1-2, except for LF and LF/HF, in week 1 , denoting a short-term increase in sympathetic activity without any other changes. This is in agreement with Hessel et al., using instrumentation methods similar to ours, that showed that MCT was associated with growth restriction, decreased mHR and increased LF/HF, in a dose-related way, that was manifested with doses as low as $30 \mathrm{mg} / \mathrm{kg}$ [17]. This may be explained not only by an autonomous nervous system deregulation, but also by direct pulmonary and cardiac MCT effects [17,33]. MCT-treated rats present an impaired response to the sympathetic nervous system as manifest by reductions of $\beta$-adrenoceptor and norepinephrine neuronal transporter densities and of norepinephrine neuronal transporter and G-protein coupled receptor kinase activity [19]. Previous observation of increased plasma noradrenaline in this experimental model further strengthens the evidence of sympathetic hyperactivity [19]. However, Akhavein et al. observed an increase of mHR in MCT injected rats [2]. This discrepancy, that still needs to be confirmed in other studies, may have been related with the use of heavier (460-495 g) male rats, not anesthetized when HR signals had been captured by telemetry, 7 weeks after a high MCT injection dose of $80 \mathrm{mg} / \mathrm{kg}$ [2]. There is no available data on non-linear HR analysis of MCT injected rats to compare with our results. However, similar changes in linear and non-linear HR indices have been observed in human pre and post natal conditions related to heart failure intrauterine growth restriction $[25,34,38]$. On the other hand, a maintenance of linear and non-linear HR indices, or an increase, namely on week 4 , was observed in the nonMCT group, except of LF/HF, denoting a long-term increase of the overall activity of linear and complex HR control systems, with a parasympathetic dominance. This may be associated to ageing and maturation and/or to behavioral reactions to the applied instrumentation conditions. This warrants further investigation in future studies, taking into account the lessons from the present study, namely on what concerns the need of special animal instrumentation methods, such as telemetry.

Indeed, although still under considerable debate [28,36], linear and non-linear HR indices are supposed to correspond to the activity of different regulatory physiologic systems [11,35]. Mean HR is likely to correspond to the intrinsic cardiac activity under the autonomic nervous system influence, estimated with the variance related linear time domain indices, namely LTI, STV, sdHR, II and $\Delta[4,8]$. HF and LF are supposed to convey discriminating information about the activity of the parasympathetic and sympathetic branches of the autonomic nervous system [27]. Entropy indices are believed to provide information about more complex HR regulatory systems presumably located in cortical central nervous centres [29]. Three recently proposed values for parameter " $r$ " were used in entropy analysis, considering the values $0.6 \mathrm{SD}, 0.9 \mathrm{SD}$ and 1.2 $\mathrm{SD}$, having in mind that the quotient between the coefficient of variation of the FHR and the coefficient of variation of the rat HR signal is about 6 [14]. These values were considered as an adjustment based on other studies performed in human fetuses [11,13], but did not evidence any significant change across the weeks of the present study. This indicates that several aspects about the mathematical approach to linear and non-linear HR analysis still need to be studied and tested. The same occurs not only regarding entropy indices, but also regarding the choice of standard spectral frequency intervals and of entropy indices, mostly derived from different experimental protocols [7,18].

As more extensively discussed in a previous paper [14], nonlinear SD1 and SD2 HR indices derived from Poincare plots [26] and scaling indices such as fractal dimension and short- and longterm correlation [3] could also had been used in this study, as the scaling behavior of HR seems to be selectively related to the alfa-adrenergic activity whereas entropy appears to be related to cholinergic activity [3]. However, those indices were not under the scope of this study, which was designed, as our last series of studies on FHR [11-14], regarding a more in-depth entropy analysis, considering different scales and a more comparable analysis with other FHR studies published in the literature [21]. Based on the results obtained using the recently proposed values for entropy parameter " $r$ " [14], the similar behavior across the weeks of protocol that was found both in the non-MCT and MCT groups, strongly justifies further research regarding the understanding of the physiological aspects associated to this set of values, which seem to correspond to a single dimension.

Putting together all the above considerations about the strengths and weaknesses of this study it may be concluded that studies on long-term HR dynamics should be performed in very strictly controlled experimental settings, as significant weekly changes may occur, both among anesthetized MCT-treated and non-treated rats, even when apparently standardized procedures are undertaken. In our study, an overall weekly maintenance or decrease of HR indices was manifest in the MCT group, except initially for LF and LF/HF, denoting a short-term increase in sympathetic activity without any other changes. On the other hand, a maintenance or an increase of HR indices was observed in the non-MCT group, namely in week 4, except for $\mathrm{LF} / \mathrm{HF}$, denoting a long-term increase of the overall activity of HR control systems, with a parasympathetic like dominance. These results suggest that linear and non-linear indices may help to characterize heart rate dynamics in chronic animal models. Further studies are warranted in this field.

\section{Acknowledgment}

This study was supported by project POSI/CPS/40153/2001 from Fundação Para a Ciência e Tecnologia, Portugal.

\section{Conflict of interest}

None.

\section{References}

[1] ACOG, 2005. American College of Obstetricians and Gynecologists. ACOG Practice Bulletin. Clinical Management Guidelines for Obstetrician-Gynecologists, Number 70, December 2005. Obstet Gynecol 106(6):1453-60. 
[2] Akhavein F, St.-Michel EJ, Seifert E, Rohlicek CV. Decreased left ventricular function, myocarditis, and coronary arteriolar medial thickening following monocrotaline administration in adult rats. J Appl Physiol 2007;103(1):287-95.

[3] Beckers F, Verheyden B, Aubert AE. Aging and nonlinear heart rate control in a healthy population. Am J Physiol Heart Circ Physiol 2006;290(6):H2560-70.

[4] Bernardes J, Costa-Pereira A. A more objective fetal heart rate baseline estimation. Br J Obstet Gynaecol 1996;103:714-5.

[5] Bernardes J, Gonçalves H, Ayres-de-Campos D, Rocha AP. Linear and complex heart rate dynamics vary with sex in relation to fetal behavioural states. Early Hum Dev 2008;84:433-9.

[6] Bruijnzeel AW, Stam R, Croiset G, Wiegant GM. Long-term sensitization of cardiovascular stress responses after a single stressful experience. Physiol Behav 2001;73(1-2):81-6.

[7] Cerutti C, Gustin MP, Paultre CZ, Lo M, Julien C, Vincent M, et al. Autonomic nervous-systempulmonary-edema and cardiovascular variability in rats-a spectral-analysis approach. Am J Physiol 1991;261(84):H1292-9.

[8] Court DJ, Parer JT, Nathanielsz PW, Parer JT.Experimental studies of fetal asphyxia and fetal heart rate interpretation Research in Perinatal Medicine. New York: Perinatology Press; 1984. p. 113-69.

[9] Dudewicz E, Mishra S. Modern mathematical statistics. Jonh Wiley and Sons, Inc; 1988.

[10] Falcao-Pires I, Gonçalves N, Henriques-Coelho T, Roncon-Albuquerque R, Moreira-Gonçalves D, Leite-Moreira Jr AF. Administration of Apelin modulates myocardial. expression of Apelin-APJ and improves right ventricular performance in MCT-induced pulmonary hypertension. Circulation 2008;118:S285.

[11] Gonçalves H, Rocha AP, Ayres-de-Campos D, Bernardes J. Internal versus external intrapartum foetal heart rate monitoring: the effect on linear and nonlinear parameters. Physiol Meas 2006;27(3):307-19.

[12] Gonçalves H, Rocha AP, Ayres-de-Campos D, Bernardes J. Linear and nonlinear fetal heart rate analysis of normal and acidemic fetuses in the minutes preceding delivery. Med Biol Eng Comput 2006;44(10):847-55.

[13] Gonçalves H, Bernardes J, Rocha AP, Ayres-de-Campos D. Linear and nonlinear analysis of heart rate patterns associated with fetal behavioral states in the antepartum period. Early Hum Dev 2007;83:585-91.

[14] Gonçalves H, Henriques-Coelho T, Bernardes J, Rocha AP, Nogueira A, LeiteMoreira A. Linear and nonlinear heart rate analysis in a rat model of acute anoxia. Physiol Meas 2008;29:1133-43.

[15] Henriques-Coelho T, Correia-Pinto J, Roncon-Albuquerque Jr R, Baptista MJ, Lourenco AP, Oliveira SM, et al. Endogenous production of ghrelin and beneficial effects of its exogenous administration in monocrotaline-induced pulmonary hypertension. Am J Physiol Heart Circ Physiol 2004;287(6):H2885-90.

[16] Henriques-Coelho T, Oliveira SM, Moura RS, Roncon-Albuquerque Jr R, Neves AL, Santos M, et al. Thymulin inhibits monocrotaline-induced pulmonary hypertension modulating interleukin-6 expression and suppressing p38 pathway. Endocrinology 2008;149(9):4367-73.

[17] Hessel MHM, Steendijk P, den Adel B, Schutte CI, van der Laarse A. Characterization of right ventricular function after monocrotaline-induced pulmonary hypertension in the intact rat. Am J Physiol Heart Circ Physiol 2006;291(5):H2424-30.

[18] Kuwahara M, Yayou K, Ishii K, Hashimoto S, Tsubone H, Sugano S. Power spectral analysis of heart rate variability as a new method for assessing autonomic activity in the rat. J Electrocardiol 1994;27(4):333-7.

[19] Leineweber K, Brandt K, Wludyka B, Beilfuss A, Ponicke K, Heinroth-Hoffmann $\mathrm{I}$, et al. Ventricular hypertrophy plus neurohumoral activation is necessary to alter the cardiac beta-adrenoceptor system in experimental heart failure. Circ Res 2002;91(11):1056-62.

[20] Lourenço AP, Roncon-Albuquerque Jr R, Bras-Silva C, Faria B, Wieland ], Henriques-Coelho T, et al. Myocardial dysfunction and neurohumoral activation without remodeling in left ventricle of monocrotaline-induced pulmonary hypertensive rats. Am J Physiol Heart Circ Physiol 2006;291(4):H1587-94.

[21] Magenes G, Signorini MG, Arduini D, Cerutti S. Fetal heart rate variability due to vibroacoustic stimulation: linear and nonlinear contribution. Methods Inf Med 2004;43(1):47-51

[22] Marshall BE, Longnecker DE. General anesthetics. In: Hardman JG, Gilman AG Limbird LE, editors. Goodman \& Gilman's. The pharmacological basis of therapeutics. ninth ed. New York: McGraw-Hill; 1996.

[23] Martinez WL, Martinez AR. Computational statistics handbook with MATLAB. CRC Press; 2002

[24] Miller WC, Rice DL, Kreusel RG, Bedrossian CWM. Monocrotaline model of noncardiogenic pulmonary-edema in dogs. J Appl Physiol 1978;45(6):962-5.

[25] Montenegro N, Bernardes J, Ayres-de-Campos D, Matias A, Areias JC. Monitoring of cardiac-extracardiac haemodynamics and automated fetal heart rate preceding intrauterine death. Eur J Obstet Gynecol Reprod Biol 1996;64(1):3-6.

[26] Moraru L, Cimponeriu L, Tong S, Thakor N, Bezerianos A. Characterization of heart rate variability changes following asphyxia in rats. Methods Inf Med 2004;43(1):118-21.

[27] Oppenheimer LW, Lewinsky RM. Power spectral analysis of fetal heart rate Baillieres Clin Obstet Gynaecol 1994;8(3):643-61.

[28] Parati G, Mancia G, Di Rienzo M, Castiglioni P, Taylor JA, Studinger P. Point: Counterpoint: Cardiovascular variability is/is not an index of autonomic control of circulation. J Appl Physiol 2006;101(2):676-82.

[29] Pincus S, Viscarello R. Approximate Entropy: A regularity measure for fetal heart rate analysis. Obstet Gynecol 1992;79:249-55.

[30] Pincus S. Aproximate entropy (ApEn) as a complexity measure. Chaos 1995;5:110-7.

[31] Pincus SM, Gevers EF, Robinson ICAF, VanDenBerg G, Roelfsema F, Hartman ML, et al. Females secrete growth hormone with more process irregularity than males in both humans and rats. Am J Physiol-Endocrinol Metab 1996;270(1):E107-15.

[32] Richman JS, Moorman JR. Physiological time-series analysis using approximate entropy and sample entropy. Am J Physiol Heart Circ Physiol 2000;278:H2039-49.

[33] Sanyal SN, Ono K. Derangement of autonomic nerve control in rat with right ventricular failure. Pathophysiology 2002;8(3):197-203.

[34] Signorini MG, Magenes G, Cerrutti S, Arduini D. Linear and nonlinear parameters for the analysis of fetal heart rate signal from cardiotacographic recordings. IEEE Trans Biomed Eng 2003;50:365-73.

[35] Task force 1996. Heart rate variability - standards of measurement, physiological interpretation and clinical use Circulation 93 1043-65 Task force of the European Society of Cardiology and the North American Society of Pacing and Electrophysiology.

[36] Taylor JA, Studinger P. Cardiovascular variability is/is not an index of autonomic control of circulation. J Appl Physiol 2006;101(2):690-1.

[37] Welch PD. The use of Fast Fourier Transform for the estimation of power spectra: a method based on time averaging over short, modified periodograms. IEEE Trans Audio Electroacous 1967;AU-15(2):70-3.

[38] Yum MK, Park EY, Kim CR, Hwang JH. Alterations in irregular and fractal heart rate behavior in growth restricted fetuses. Eur J Obstet Gynecol 2001;94:51-8. 